\title{
Development of composite electrode materials based on nickel oxide for additive manufacturing of fuel cells
}

\author{
Artem Bagishev ${ }^{1 *}$, Alexander Titkov ${ }^{1}$, Alexander Vorobyev $^{1}$, Tatyana Borisenko $^{1}$, Victor \\ Bessmeltsev $^{2}$, Denis Katasonov ${ }^{2}$, and Alexander Nemudry ${ }^{1}$ \\ ${ }^{1}$ Institute of Solid State Chemistry and Mechanochemistry SB RAS, Kutateladze St. 18, Novosibirsk, \\ 630128, Russia \\ ${ }^{2}$ Institute of Automation and Electrometry SB RAS, Academician Koptyug Av. 1, Novosibirsk, \\ 630090, Russia
}

\begin{abstract}
In this work, a new composition of a paste based on nanosized nickel oxide $(\mathrm{NiO})$ for jet-based 3D printing has been developed and investigated. The optimal parameters of anode paste with necessary viscosity and $\mathrm{NiO}$ loading have been found for printing by using custom made laboratory 3D printer. Experiments have been carried out to print two- and three-dimensional test objects using the developed ceramic paste; printing modes have been investigated to fabricate objects of a given shape and size with laser sintering of the deposited layers. Experiments have been conducted for the selection of optimal temperature conditions for the post-processing of the printed samples.
\end{abstract}

\section{Introduction}

Alternative methods of generating electricity have received more and more attention in recent years [1]. This interest is caused, on the one hand, by a reduction in the consumption of mineral fuels, and on the other hand, by the economic efficiency of innovative approaches to power generation and strict environmental requirements for new technologies.

One of the directions of development of alternative energy is high-temperature electrochemical devices [1,2]. Such devices allow a) to efficiently (60-70\% efficiency) convert fossil fuel and hydrogen into electricity by using solid oxide fuel cells (SOFCs) and b) to process carbon dioxide and water vapor into synthesis gas $\left(\mathrm{CO}+2 \mathrm{H}_{2}\right)$, utilizing greenhouse emissions gases, efficiently storing energy from low-cost energy sources, using high-temperature solid oxide electrolyzers (SOE) [3]. Hydrogen is not only the basis for ecologically clean hydrogen energy, but also a raw material for various technologies of hydroprocessing, large-tonnage production of ammonia and methanol, and subsequent chemical products [4].

Structurally, SOFC can be divided into flat and tubular $[1,4,5]$. The planar design is widely used in stationary devices with a capacity of megawatt and above, as it provides

\footnotetext{
*Corresponding author: artembagishev15@gmail.com
} 
good heat and mass transfer, compactness of the assembly and allows the use of standard methods of processing ceramics [6]. A significant disadvantage of the planar configuration is the high requirements for the absence of significant temperature gradients along the membrane, which can lead to the destruction of the device during thermal cycling and sharp temperature drops.

The problem can be solved by using microtubular (MT) membranes [5], the advantages of which are improved thermal and mechanical stability, ease of sealing. The rapid launch of high-temperature ECDs opens up a promising niche for compact mobile devices in transportation, military and consumer electronics applications.

A complete SOFC battery made conventional manufacturing processes usually requires many steps. All of these steps are time consuming, not easy replicable and expensive, material consuming and waste generating. Tape casting, screen printing, lamination and stacking are common procedures for making a stack of planar configuration SOFCs. In addition, a tubular SOFC stack follows the steps in which each individual cell is prepared separately and then all cells are stacked. Since there are many joints and seals in the final device, this manufacturing process greatly affects the reliability and durability of SOFC systems [1,7]. Therefore, new methods and approaches need to be developed to minimize the number of stages in the manufacture of SOFCs and, among other things, to manufacture the entire assembly at once. One of them is $3 \mathrm{D}$ printing, which allows fabricating complex $3 \mathrm{D}$ objects layer by layer from computer 3D model using a special software (CAD) $[8,9]$.

Freeform object manufacturing technologies such as 3D printing have only recently demonstrated their suitability for the manufacture of highly complex dense ceramic parts with good mechanical properties $[10,11]$. In particular, the possibility of 3D printing of an electrolyte-supporting cell of SOFC of complex shape was demonstrated, which gave an increase in power by about $50 \%$. [10].

In this work, we have developed and investigated the composition of paste for 3D printing. Using the prepared paste, we have successfully printed test $3 \mathrm{D}$ objects by custom made laboratory $3 \mathrm{D}$ printer and investigated its structure and morphology after printing and post processing.

\section{Experimental}

Nickel oxide (SOFCMAN, China), 2-Butoxyethanol (BG, Vekton, Russia), BYK (BYKChemie GmbH, Germany), polyvinyl butyral (PVB, Acros organics, US), dibutyl phthalate (DBF, Acros organics, US) were used to synthesize the powders.

Paste formulation and printing. The powder was preliminarily milled using an APS grinding system using bead balls $(\mathrm{d}=1.2 \mathrm{~mm})$ in ethanol. For a paste preparation BYK (loading is $8 \mathrm{wt} \%$ of $\mathrm{NiO}$ ), DBF and PVB ( $5 \mathrm{wt} \%$ of BG) were dissolved in BG, placed in the APS grinding system with grinding balls (diameter $1.2 \mathrm{~mm}$ ), then the $\mathrm{NiO}$ powder was added and stirred at $4000 \mathrm{rpm}$ for 2 hours, then the paste was separated from the balls.

For printing, a laboratory 3D printer was used custom made by Institute of Automation and Electrometry SB RAS. The printer combines the ability to use different low and high viscous injection systems for direct jet-based printing different materials and a laser processing system based on fiber laser. The printer has a three-dimensional heated table $(10 \times 10 \times 15 \mathrm{~cm})$ for the formation of two-dimensional and three-dimensional objects. All printing experiments were performed using a pneumatic micro-metering valve and a 0.25 mm nozzle (Nordson Corporation, Germany-USA). Laser sintering of the printed layers using a fiber laser operating at a wavelength of $1.064 \mu \mathrm{m}$ in pulse-periodic mode was performed in one pass regime with changeable laser power. The laser pulse duration and frequency were $4 \mathrm{~ns}$ and $250 \mathrm{kHz}$, respectively; the average power changed in the range of $0.45-2.7 \mathrm{~W}$. Exposure was carried out using the raster scanning of squares $5 \times 5,10 \times 10 \mathrm{~mm}^{2}$. 
The raster spacing was $0.0125 \mathrm{~mm}$, the size of the focused laser beam was $0.035-0.05 \mathrm{~mm}$ in terms of intensity $\mathrm{e}^{-2}$, and the exposure time was $0.15-0.2 \mathrm{~ms}$.

Characterization. The viscosity of pastes was measured using a Brookfield DV3T-RV viscometer (Brookfield Engineering Labs Inc., USA) in cone / plate geometry at $25^{\circ} \mathrm{C}$. A study of the samples by scanning electron microscopy (SEM) was performed using a Hitachi $3400 \mathrm{~N}$ scanning electron microscope (Hitachi Ltd., Japan). Microsizer 201(Microsizer, Russia) was used for the particle size distribution analysis.

\section{Results and discussion}

The selection of the optimal parameters of the pastes was carried out by varying the amount of polymer binder (PVB+DBF), the amount of composite powder in all pastes was constant and equal to 60 weight percent (average particle size was $1.38 \mu \mathrm{m}$ ). The viscosity of pastes depends on the amount of polymer binder in the composition, so series of pastes have been prepared with 5, 10 and 15 percent of the polymer binder. At a shear rate of $1 \mathrm{~s}^{-1}$, the paste with $10 \%$ polymer binder has a viscosity of $4000 \mathrm{cP}$, and at a shear rate of $10 \mathrm{~s}^{-1} 600 \mathrm{cP}$ (Fig. 1a), paste with $15 \%$ polymer binder at $1 \mathrm{~s}^{-1}$ has a viscosity of $25000 \mathrm{cP}$, at shear rate $10 \mathrm{~s}^{-1}-1500 \mathrm{cP}$ (Fig. 1b).
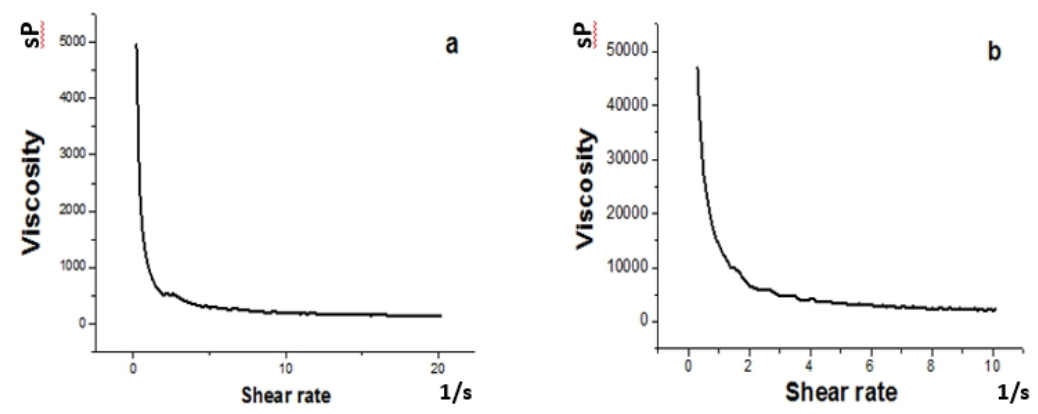

Fig.1. Viscosities of pastes at various concentrations of the polymer binder a) $10 \%$ polymer binder, b) $15 \%$ polymer binder.

To start printing 3D objects, the optimal drop was firstly selected $\left(\mathrm{d}_{\mathrm{drops}}=0.5 \mathrm{~mm}\right.$, ideal round shape after drying). For this, test printing is performed with different modes of frequency and time of opening the printhead valve. The optimal parameters for the working paste are $20 \mathrm{~Hz}$ - valve opening frequency and $700 \mathrm{~ms}$ valve opening time. An array of printed dots using epy optimal parameters is presented on Fig. 2.

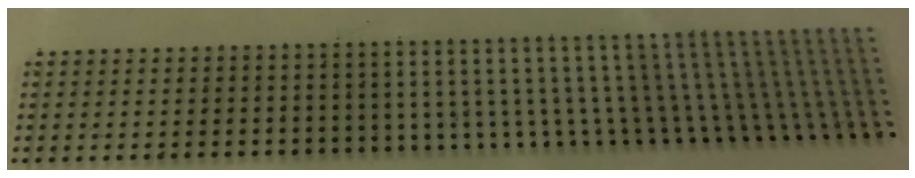

Fig.2. The array of printed dots using optimal parameters $\left(\mathrm{d}_{\mathrm{drops}}=0.5 \mathrm{~mm}\right.$, ideal round shape after drying).

Next, the model of the printed object was loaded into the computer program, it was sliced into layers and the object was printed directly. Ten layers sequentially printed and subjected to a laser treatment. Laser was selectively scanned the printed pattern. The photo and SEM images of printed object are presented on the Fig. 3. Geometrical parameters of 
the printed object: length $-10 \mathrm{~mm}$, width $-15 \mathrm{~mm}$, height $3 \mathrm{~mm}$. As can be seen on Fig. $3 \mathrm{~b}-\mathrm{d}$, the printed bar has a porous and layered structure. The grain size is about 1-2 $\mu \mathrm{m}$. The thickness of one layer after printing is about 50 microns. Interlayer pores are closed to spherical, not connected to each other, the pore diameter varies from 5-20 microns. Most likely, the formation of porosity occurs due to the excessive pressure of gaseous products appearing during laser processing and partial decomposition of the organic components of the paste, which affect the surface of the object.

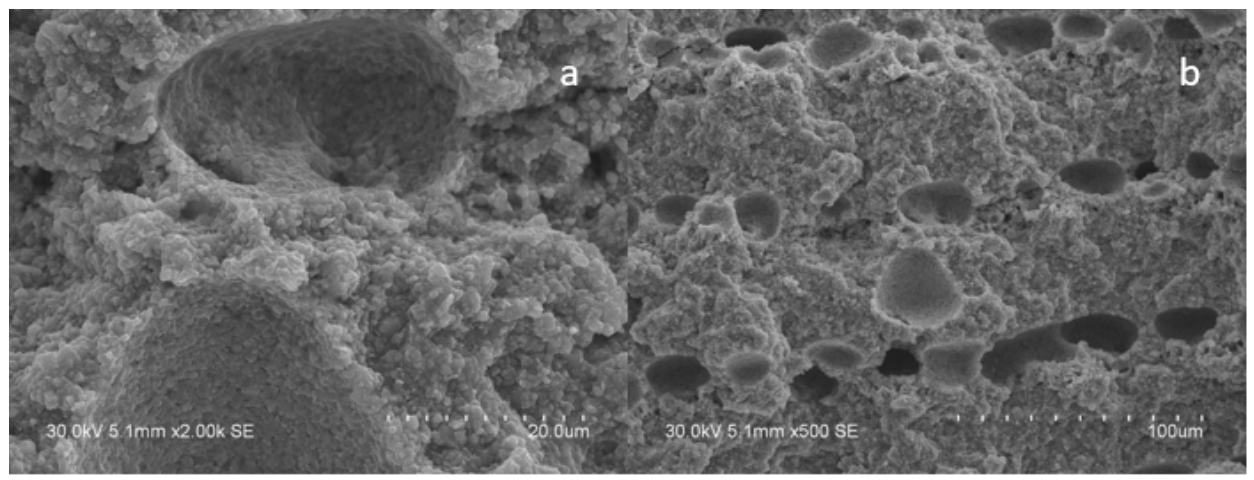

Fig.3. SEM images of the microstructure of the printed object - top view (a)-(b).

After being printed the test object was sintered in a high-temperature PVC 1.6 furnace The optimum temperature sintering for SOFC anode billets is $1400^{\circ} \mathrm{C}$ [12] for 11 hours in an air atmosphere, at temperatures below, the formation of a dense sintered structure of the object did not occur. Figure 4 shows a comparison of the object after sintering, in which we see that after thermal, the particles are baked together to form a dense structure. After sintering, we observe a violation of the sphericity of the pores, the coalescence of a large number of pores. During sintering, the entire object shrinks, the layered structure becomes less pronounced. Shrinkage after sintering for all geometrical dimensions was found to be around $30 \%$.

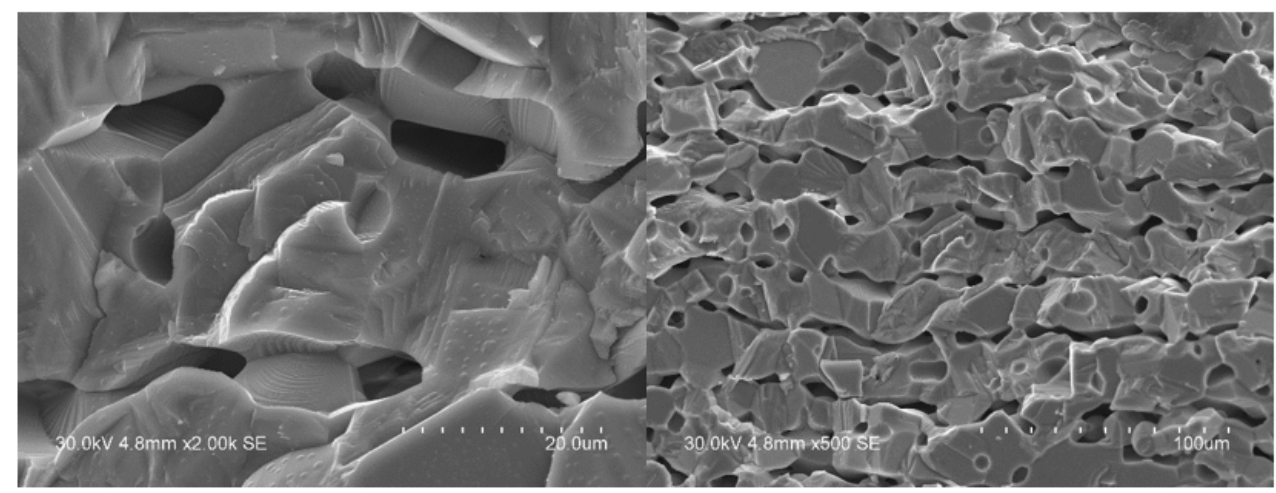

Fig.4. SEM images of the microstructure of the annealed object.

Thus, it has been shown that, using injection printing and laser processing, it is possible to form layer-by-layer 3D objects from a paste with a filler $\mathrm{NiO}$. In this case, laser treatment makes it possible to partially remove the organic component and partially sinter the $\mathrm{NiO}$ particles together, as a result it is possible to obtain a layer-by-layer object. Using the thermal post-processing, strong sintered ceramic object can be formed with a defined $3 \mathrm{D}$ computer model of the shape, taking into account the shrinkage during sintering. 
Further research is needed to quantify mechanical properties and their relationship to printing conditions.

This work is supported by the Russian Science Foundation under grant (No. 21-79-30051).

\section{References}

1. Seventh Ed. Fuel Cell Handbook (US, Pittsburgh, 2004)

2. L. Umberto, Renew. Sustain. Energy Rev. 30, 164 (2004)

3. J.P. Stempien, Q. Sun, S.H. Chan, J. Power Technol. 93, 216 (2013)

4. F. Ramadhani, M. Hussain, H. Mokhlis, S. Hajimolanda, Renew. Sustain. Energy Rev. 76, 460 (2017)

5. Q. Hodjati-Pugh, A. Dhir, R. Steinberger-Wilckens, Appl. Sci. 11, (2021)

6. Z. Zakaria, M.A. Mat, S. Abu Hassan, Y.B. Kar, Int. J. Energy Res. 44, 594 (2019)

7. Y.J. Kim et al., Int. J. Hydrog. Energy 45, 19143 (2020)

8. Z. Chen et al., J. Eur. Ceram. Soc. 39, 661(2019)

9. H.W. Tan, T. Tran, C.K. Chua, Virtual Phys. Prototyp. 11, 271 (2016)

10. A. Pesce et al., J. Mater. Chem. A 8, 16926 (2020)

11. Z. Chen et al., J. Adv. Ceram. 10, 195 (2021)

12. A. Bagishev et al., Chem. Sustain. Develop. 27, 275 (2019) 